\title{
Dysregulated $\mathrm{ICOS}^{+}$proinflammatory and suppressive regulatory $\mathbf{T}$ cells in patients with rheumatoid arthritis
}

\author{
HONG-XIA WANG ${ }^{1 *}$, XIA KANG ${ }^{1,2^{*}}$, SHUAI CHU ${ }^{1 *}$, HAIXIA LI $^{1}, \mathrm{XIN} \mathrm{LI}^{1}$, \\ XIAOFENG YIN ${ }^{1}$, YU-RONG QIU ${ }^{1}$ and WEINAN LAI ${ }^{3,4}$ \\ ${ }^{1}$ Laboratory Medicine Center, Nanfang Hospital, Southern Medical University, Guangzhou, Guangdong 510515; \\ ${ }^{2}$ Department of Clinical Laboratory, Nanlou Division, Chinese PLA General Hospital, National Clinical Research Center \\ for Geriatric Diseases, Beijing 100853; ${ }^{3}$ Department of Rheumatology and Immunology, Nanfang Hospital, \\ Southern Medical University, Guangzhou, Guangdong 510515, P.R. China; ${ }^{4}$ Division of Rheumatology, \\ University of Washington Medical Center, Seattle, WA 98109, USA
}

Received February 4, 2018; Accepted July 20, 2018

DOI: $10.3892 /$ etm.2018.6657

\begin{abstract}
Regulatory T cells (Tregs) serve an important role in the pathogenesis of rheumatoid arthritis (RA) by regulating autoimmunity and inflammation. Humans and mice contain inducible T-cell costimulator-positive $\left(\mathrm{ICOS}^{+}\right)$Tregs, although their role in RA is unclear. A total of 33 patients with RA and 17 normal control (NC) subjects were examined. The proportion of $\mathrm{ICOS}^{+}$Tregs in the peripheral blood and intracellular cytokine levels in these cells were assessed using flow cytometry. The percentage of $\mathrm{ICOS}^{+}$Tregs increased in the cohort of patients with RA compared with the NCs. Such increases were much larger in patients with inactive RA compared with patients with active RA. Additionally, ICOS ${ }^{+}$ Tregs expressed multiple suppressive cytokines, including interleukin (IL)-10, transforming growth factor- $\beta$ and IL-35, but expressed low levels of IL-17. Importantly, the expression of suppressive cytokines in $\mathrm{ICOS}^{+}$Tregs from patients with active RA decreased, but IL-17 expression noticeably increased compared with patients with inactive RA. The present findings suggested that $\mathrm{ICOS}^{+}$Tregs may perform inflammatory and inhibitory functions, and abnormal $\mathrm{ICOS}^{+}$Tregs numbers and functions may contribute to the pathogenesis of RA.
\end{abstract}

Correspondence to: Dr Yu-Rong Qiu, Laboratory Medicine Center, Nanfang Hospital, Southern Medical University, 1838 North Guangzhou Avenue, Guangzhou, Guangdong 510515, P.R. China E-mail: qiuyuronggz@126.com

Dr Weinan Lai, Department of Rheumatology and Immunology, Nanfang Hospital, Southern Medical University, 1838 North Guangzhou Avenue, Guangzhou, Guangdong 510515, P.R. China

E-mail: laiwn@uw.edu

${ }^{*}$ Contributed equally

Key words: inducible T-cell costimulator-positive regulatory $\mathrm{T}$ cells, rheumatoid arthritis, cytokines

\section{Introduction}

Rheumatoid arthritis (RA) is a systemic, chronic, inflammatory autoimmune disease that affects joints and other tissues, including the skin and kidneys (1). RA affects a large number of individuals worldwide, with nearly three times as many females as men being diagnosed with RA (2). Studies have suggested that the pathogenesis of RA is triggered by failure in the maintenance of peripheral tolerance to self-antigens, which leads to the activation and expansion of autoreactive $\mathrm{T}$ and $\mathrm{B}$ cells $(3,4)$. These pathogenic T cells and autoantibodies in turn cause joint inflammation that eventually leads to irreversible articular cartilage damage as well as the breakdown of osseous tissue $(3,4)$. Various proinflammatory cytokines serve important roles in RA development (4). Patients with RA exhibit increased numbers of interleukin (IL)-17-producing $\mathrm{T}$ helper (h)17 cells in the peripheral blood in comparison with healthy subjects $(5,6)$. Additionally, IL-17 was elevated in the synovial fluid of patients with RA and was involved in the pathogenesis of RA (7,8). Regulatory T cells (Tregs) were revealed to be crucial for peripheral self-tolerance, as they actively suppress auto-reactive lymphocytes as well as other immune cells and express suppressive cytokines, including IL-10 and transforming growth factor (TGF)- $\beta$ (9). It has been reported that Tregs serve an important role in the pathogenesis of RA by regulating autoimmunity and inflammation $(10,11)$. Inducible T-cell costimulator (ICOS) is a co-receptor belonging to the cluster of differentiation (CD)28 immunoglobulin (Ig) super family that provide co-stimulatory signals to $\mathrm{T}$ cells during antigen-mediated activation (12).

Reports on murine models for autoimmune diabetes and allergic asthma have described the important roles of ICOS in the generation, maintenance and suppressive functions of Tregs $(13,14)$. Notably, ICOS ${ }^{+}$Tregs exhibited a stronger suppressive activity compared with ICOS $^{-}$Tregs (15). Furthermore, $\mathrm{ICOS}^{+}$Tregs released IL-10 to suppress dendritic cell function and TGF- $\beta$ to suppress T cell function, while ICOS Tregs mainly secreted TGF- $\beta$ (16). The blockade or absence of ICOS inhibited the production of IL-10 and abrogated the inhibitory function of Tregs $(17,18)$. Although an increasing 
number of studies have indicated that ICOS signaling is necessary for Treg suppression in various diseases $(13,14)$, little is known about the roles of ICOS $^{+}$Tregs in RA. Various studies have reported that a Th17/Treg imbalance is associated with the development of RA (19-21). However, the precise association between $\mathrm{ICOS}^{+}$Tregs and Th17 in patients with RA has yet to be determined. The current study examined the $\mathrm{ICOS}^{+}$ Tregs in a cohort of Chinese patients with RA, particularly in patients with inactive RA (iRA). The proportions of $\mathrm{ICOS}^{+}$ Tregs in patients with RA were assessed according to DAS28 scores. The current study also explored the expression levels of $\mathrm{ICOS}^{+}$Tregs from patients with iRA and the levels of suppressive cytokines, including IL-10, TGF- $\beta$, IL-35 and IL- 17 . The present study was performed to clarify whether ICOS $^{+}$Tregs may perform inflammatory and inhibitory functions, and if abnormal $\mathrm{ICOS}^{+}$Tregs numbers and functions may contribute to the pathogenesis of RA.

\section{Patients and methods}

Patients and healthy controls. The current study, which took place at the Department of Rheumatology of Nanfang Hospital, Southern Medical University (Guangzhou, China) from January 2015 to January 2016, examined 33 Chinese patients diagnosed with RA according to the American College of Rheumatology (ACR) criteria (22). These patients included 31 females and 2 males (age, 49.76 \pm 1.73 years). Information concerning the patient's medical history, number of classification criteria fulfilled and laboratory findings were obtained at the time of the clinic visit. Whole blood samples were collected and disease activity was measured using the 28-joint disease activity index (DAS28) (23). Based on the DAS28, 10 patients possessed iRA (DAS28 score $\leq 3.3$ ) and 23 possessed aRA (DAS28 score $>3.3$ ) during the study. Additionally, 17 healthy subjects were recruited to serve as the normal control (NC) group, the details of which are presented in Table I. All participants provided written consent during their enrollment. The Ethics Committee of Nanfang Hospital approved the current study.

Antibodies and reagents. Anti-CD4-fluoresceinisothiocyanate (FITC; cat. no. 11-0048-42), anti-ICOS-phycoerythrin (PE)-Cy7 (cat. no. 85-25-9948-42) and anti-TGF- $\beta$-PE (cat. no. 12-9829-42) antibodies were purchased from (eBioscience; Thermo Fisher Scientific, Inc., Waltham, MA, USA). Anti-CD25-PerCP-Cy5.5 (cat. no. 560503), Mouse IgGl-PE-Cy7 (cat. no. 25-4714-41), Mouse IgGl-PE (cat. no. 12-4714-41), Mouse IgGl-PerCP-Cy5.5 (cat. no. 550795), anti-Foxp3-PE-CF594(cat.no.562421),Mouse IgGl-PE-CF594 (cat. no. 562292), anti-IL-10-APC (cat. no. 554707) and anti-IL-17-V450 (cat. no. 560610), Mouse IgGl-V450 (cat. no. 560373) antibodies were purchased from BD Biosciences (Franklin Lakes, NJ, USA). Anti-human-EBI3-APC (cat. no. IC6456A), anti-human-p35-PE (cat. no. IC2191P) antibodies were purchased from R\&D Systems, Inc. (Minneapolis, MN, USA). Additional reagents included Mouse IgGl-APC (cat. no. 70-CMG105-10; MultiSciences, ZheJiang, China), OptiLyse C lysing solution (Beckman Coulter, Inc., Brea, CA, USA), PBS, fetal bovine serum (FBS; both Thermo Fisher Scientific, Inc.), Foxp3 Staining
Buffer Set kit (BD Biosciences, NJ, USA) and Monensin (eBioscience; Thermo Fisher Scientific, Inc.), phorbol myristate acetate (PMA) (cat. no. P-8139; Sigma-Aldrich; Merck KGaA, Darmstadt, Germany) ionomycin (cat. no. I-0634; Sigma-Aldrich; Merck KGaA), brefeldin A (BFA; cat. no. B-7651; Sigma-Aldrich; Merck KGaA) and Ficoll (Tianjin Haoyang 4E Products Technology Co., Ltd., Tianjin, China).

Flow cytometry. For fresh staining, $100 \mu \mathrm{l}$ fresh heparinized peripheral blood was initially stained with anti-CD4-FITC, anti-CD25-PerCP-Cy5.5, anti-ICOS-PE-Cy7, and the aforementioned isotypes (Mouse IgGl-PE-Cy7 and Mouse IgGl-PerCP-Cy5.5; all at a dilution of 1:20) for $20 \mathrm{~min}$ at $4{ }^{\circ} \mathrm{C}$ following the lysis of red blood cells using OptiLyse $\mathrm{C}$ lysing solution. The cells were washed twice with $1 \mathrm{ml}$ washing buffer (PBS with 2\% FBS) and the Foxp3 Staining Buffer Set kit was used to perform intracellular staining of Foxp3 according to the manufacturer's protocol. Acquisitions were performed on a BD LSRFortesa flow cytometer (BD Biosciences), collecting $\geq 0.1$ million events for each sample. The data was analyzed with BD FACSDiva software v8.0.2. (BD Biosciences).

Intracellular cytokine detection. To detect intracellular cytokines, including IL-10, IL-17, TGF- $\beta$ and IL-35 fresh peripheral blood cells were stimulated with PMA $(40 \mathrm{ng} / \mathrm{ml})$ and ionomycin $(1 \mu \mathrm{g} / \mathrm{ml})$ in the presence of BFA $(20 \mu \mathrm{g} / \mathrm{ml})$ at $37^{\circ} \mathrm{C}$ for $5 \mathrm{~h}$. Following stimulation, cells were collected and stained for anti-CD4-FITC, anti-CD25-PerCP-Cy5.5, anti-ICOS-PE-Cy7 and indicated intracellular cytokines such as anti-Foxp3-PE-CF594, anti-TGF- $\beta$-PE, anti-IL-10-APC, anti-IL-17-V450, anti-human-EBI3-APC and anti-human-p35-PE (all 1:20) respectively. Samples were collected using a BD LSRFortesa flow cytometer and data was analyzed using BD FACSDiva software v8.0.2.

Peripheral blood mononuclear cells culture and cytokine analysis. Ficoll density gradient centrifugation $(400 \mathrm{x} \mathrm{g}$, $30 \mathrm{~min}, 21^{\circ} \mathrm{C}$ ) was used to isolate fresh peripheral mononuclear blood cells (PBMCs) from $5 \mathrm{ml}$ heparin-treated venous blood samples. PBMCs were cultured for 6 days in 12-well culture plates pre-coated with $1 \mu \mathrm{g} / \mathrm{ml}$ purified NA/LE Mouse Anti-Human CD3 (cat. no. 555336; BD Pharmingen; BD Biosciences) with the addition of 300 U/ml recombinant human IL-2 (cat. no. 200-02; Perprotech, Inc., London, UK) for T-cell receptor (TCR) stimulation. Aliquots of PBMCs $\left(1 \times 10^{6}\right)$ in $2 \mathrm{ml}$ RPMI 1640 (cat. no. 72400047; Gbico; Thermo Fisher Scientific, Inc.) were supplemented with $10 \%$ heat-inactivated fetal bovine serum (Gbico; Thermo Fisher Scientific, Inc.), glutamine (2 mM), penicillin $(200 \mathrm{U} / \mathrm{ml})$ and streptomycin $(100 \mu \mathrm{g} / \mathrm{ml})$. Cells were either stained and analysis or treated with PMA and ionomycin in the presence of BFA for $5 \mathrm{~h}$ prior to harvesting, performing intracellular cytokine staining and conducting fluorescence-activated cell sorting analysis.

Statistical analysis. All statistical analyses were performed using Prism v5.0 GraphPad software (GraphPad Software, Inc., La Jolla, CA, USA). Quantitative data were expressed 
Table I. Demographic features of normal controls and RA patients.

\begin{tabular}{lcc}
\hline Variable & $\mathrm{RA}$ & $\mathrm{NC}$ \\
\hline Total number & 33 & 17 \\
Sex (male/female) & $2 / 31$ & $1 / 16$ \\
Age (years) & $49.76 \pm 1.73$ & $33.35 \pm 1.39$ \\
DAS28 $>3.3$ & 23 & $\mathrm{~N} / \mathrm{A}$ \\
DAS $28 \leq 3.3$ & 10 & $\mathrm{~N} / \mathrm{A}$ \\
\hline
\end{tabular}

Ages are presented as mean \pm standard error of the mean. Inactive RA is defined as DAS28 score $\leq 3.3$, active RA is defined as DAS28 score >3.3. RA, rheumatoid arthritis; NC, normal control; DAS28, 28-joint disease activity index; N/A, not available.

as mean \pm standard error of the mean. One-Way analysis of variance followed by a Kruskal-Wallis $\mathrm{H}$ test was performed to compare three groups. Two-group comparisons were examined by a Mann-Whitney U test. Spearman's correlation was used to assess correlations. $\mathrm{P}<0.05$ indicated that the difference between groups was statistically significant.

\section{Results}

$I C O S^{+}$Tregs frequencies are higher in patients with RA but negatively correlate with disease activity. Tregs are essential for the maintenance of peripheral tolerance, for preventing autoimmunity and for limiting chronic inflammatory diseases (24). Although it has been reported that the number of Tregs are decreased in patients with RA (3) and $\mathrm{ICOS}^{+}$Tregs may be involved in the pathogenesis of numerous tumors and autoimmune diseases (9), it is unknown whether or not $\mathrm{ICOS}^{+}$ Tregs areassociated withRA. Toaddress this issue, the percentages of ICOS $^{+}$Tregs identified in a cohort of Chinese patients with RA and NCs were evaluated. $\mathrm{ICOS}^{+}$Tregs percentages were significantly increased in patients with RA compared with the NCs $(6.46 \pm 0.56 \%$ vs. $2.15 \pm 0.20 \%$; $\mathrm{P}<0.0001$; Fig. $1 \mathrm{~A}$ and B). By contrast, the percentages of ICOS- Tregs in patients with RA were considerably decreased $(34.77 \pm 2.10 \%$ vs. $58.08 \pm 2.40 \%$; $\mathrm{P}<0.0001$; Fig. 1C). Patients with RA were further divided into aRA and iRA groups according to the DAS28 scores. As indicated in Fig. 1D, the percentages of $\mathrm{ICOS}^{+}$Tregs were significantly increased in the aRA group $(4.60 \pm 0.23 \% ; \mathrm{P}<0.001)$ and the iRA group $(10.74 \pm 0.73 \%$; $\mathrm{P}<0.001)$ compared with the NC group $(2.15 \pm 0.20 \%)$. Additionally, the iRA group exhibited twice the number of ICOS $^{+}$Tregs as the aRA group $(\mathrm{P}<0.0001)$. Notably, DAS28 scores and ICOS $^{+}$Tregs percentages were negatively correlated in patients with RA $(r=-0.681 ; \mathrm{P}<0.001$; Fig. 1E). Thus, ICOS $^{+}$Tregs were elevated in patients with RA; however, the magnitude of increase was negatively correlated with disease activity in all patients.

$I C O S^{+}$Tregs/Th17 ratios are disturbed in patients with $R A$. Th17 cells serve important roles in autoimmune inflammation (25). Consistent with previous studies $(5,6)$, it was revealed that Th17 cell percentages in the patients with RA were significantly larger compared with the NCs
(6.04 $\pm 0.84 \%$ vs. $0.43 \pm 0.05 \%$; $\mathrm{P}<0.0001$; Fig. $2 \mathrm{~A})$. Additionally, Th17 percentages were $\sim 6.5$ times higher in patients with aRA $(8.09 \pm 0.91 \%)$ compared with that in patients with iRA $(1.34 \pm 0.18 \% ; \mathrm{P}<0.0001)$ and $\sim .5$ times higher than in the NCs $(0.43 \pm 0.05 \% ; \mathrm{P}<0.0001 ;$ Fig. $2 \mathrm{~B})$. Although the number of Th17 cells was significantly lower in the iRA group compared with that in the aRA group, Th17 percentages in the iRA group were remained significantly high compared with the NCs $(\mathrm{P}<0.0001)$.

It has been reported that Tregs can inhibit Th17 activity, and a skewed Th17/Treg balance may be a characteristic feature of chronic inflammatory diseases such as RA (21). However, the association between $\mathrm{ICOS}^{+}$Tregs and Th17 in RA remains controversial. Given the increases of $\mathrm{ICOS}^{+}$Tregs and Th17 cells in patients with RA, their relative abundance in patients with RA and NCs were analyzed. The ratios between $\mathrm{ICOS}^{+}$ Tregs and Th17 cells were significantly lower in the aRA group $(0.97 \pm 0.19)$ compared with the NCs $(6.07 \pm 0.77 ; \mathrm{P}<0.001)$. However, the ratios were significantly higher in the iRA group $(10.17 \pm 1.99)$ in comparison with either the aRA group $(0.97 \pm 0.19 ; \mathrm{P}<0.0001)$ or the NCs $(6.07 \pm 0.77 ; \mathrm{P}<0.05 ;$ Fig. $2 \mathrm{C})$.

Taken together, these data reveal that $\mathrm{ICOS}^{+}$Tregs and Th17 were increased in patients with RA; however, their respective increases differed depending on the status of the disease. This suggests that imbalances between $\mathrm{ICOS}^{+}$Tregs and Th17 may contribute to disease progression and remission.

Anti-inflammatory cytokines increase in $R A$, particularly $i R A$, whereas pro-inflammatory cytokine $I L-17$ increases in $a R A$. Cytokines produced by various synovial cell populations serve significant roles in the pathogenesis of RA (26). To further examine whether cytokine profiles of ICOS $^{+}$Tregs were dysregulated in patients RA, T cells were stimulated with PMA and ionomycin for $5 \mathrm{~h}$ in the presence of BFA, followed by intracellular staining to detect IL-10, TGF- $\beta$, IL-35 (anti-inflammatory cytokines) and IL-17 (a pro-inflammatory cytokine). As demonstrated in Fig. 3A and B, ICOS ${ }^{+}$ Tregs from the iRA group contained twice the number of IL-10-producing cells than those from the aRA group $(9.75 \pm 1.32 \%$ vs. $4.09 \pm 0.32 \% ; \mathrm{P}<0.005)$ and 5 times more IL-10-producing cells compared with those from the NCs $(1.90 \pm 0.59 \% ; \mathrm{P}<0.0005)$. The percentages of IL-10-producing $\mathrm{ICOS}^{+}$Tregs from patients with aRA were higher compared with those in NCs $(\mathrm{P}<0.005)$.

Similarly, the percentages of TGF- $\beta$-producing ICOS $^{+}$ Tregs were increased in patients with iRA in comparison with patients with aRA $(13.25 \pm 0.89 \%$ vs. $5.52 \pm 0.43 \% ; \mathrm{P}<0.0001)$ and the NCs $(7.58 \pm 1.93 \%$; $\mathrm{P}<0.05$; Fig. $3 \mathrm{C}$ and D). However, no significant differences in TGF- $\beta$-producing $\mathrm{ICOS}^{+}$Tregs were identified between the aRA group and the NCs.

IL-35, which serves a role in immune suppression of murine Treg, is a dimeric protein composed of IL-12 $\alpha$ and IL-27 $\beta$ chains that is encoded by IL12A and EBI3 genes, respectively (27). IL-35 producing ICOS $^{+}$Tregs were detected from fresh PBMCs after $5 \mathrm{~h}$ of PMA and ionomycin stimulation. However, as Fig. 3E and $\mathrm{F}$ demonstrated, after stimulation of PBMCs with anti-CD3 and IL-2 in vitro for 6 days, IL-35-producing cells could be detected in $\mathrm{ICOS}^{+}$Tregs and were significantly increased in the iRA group in comparison with the aRA group $(9.74 \pm 0.65 \%$ vs. $3.57 \pm 0.53 \%$; $\mathrm{P}<0.0001)$. The NCs exhibited a small number of IL-35-producing ICOS ${ }^{+}$ 
A

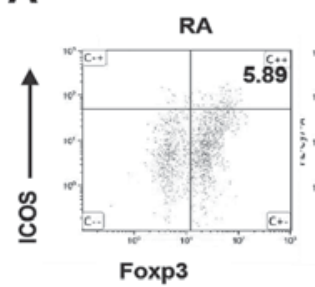

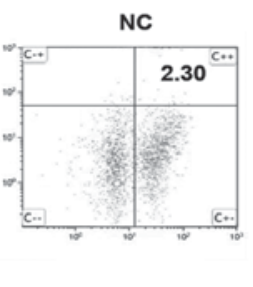

B

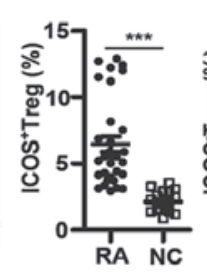

C

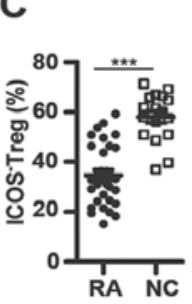

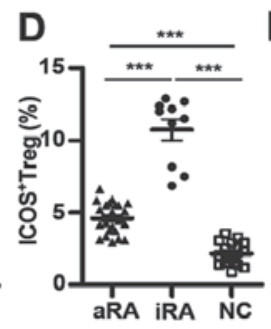

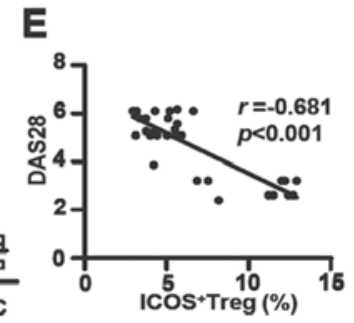

Figure 1. ICOS ${ }^{+}$Tregs frequencies are higher in patients with RA, but negatively correlate with disease activity. (A) Representative dot plots of Foxp3 and ICOS staining in the gating of cluster of differentiation $4^{+}$lymphocytes from patients with RA and NCs. The percentage of (B) ICOS ${ }^{+}$Tregs and (C) ICOS Tregs $^{-}$ in patients with RA $(n=33)$ and NCs $(n=17)$. (D) The percentage of ICOS ${ }^{+}$Tregs from patients with aRA $(n=23)$, patients with iRA $(n=10)$ and NCs $(n=17)$. (E) The correlation between the percentages of ICOS $^{+}$Tregs from patients RA with the DAS28 score. Data are presented as mean \pm standard error of the mean. ${ }^{* * *} \mathrm{P}<0.0001$. ICOS, inducible T-cell costimulator; RA, rheumatoid arthritis; NCs, normal controls; aRA, active RA; iRA, inactive RA; Tregs, regulatory T cells; DAS28, 28-joint disease activity index.

Tregs in comparison with the aRA group $(\mathrm{P}<0.0001) \mathrm{ICOS}^{+}$ Tregs from the NCs $(0.17 \pm 0.07 \%)$ possessed significantly fewer IL-35-producing cells compared with those from the iRA group $(\mathrm{P}<0.0005)$.

In contrast to these suppressive cytokines, the aRA group contained higher percentages of IL-17-producing cells within $\mathrm{ICOS}^{+}$Tregs compared with the iRA group $(11.23 \pm 0.79 \%$ vs. $2.89 \pm 0.31 \%$, respectively) or the NCs (2.22 $\pm 0.58 \%$; both $\mathrm{P}<0.0001$; Fig. $3 \mathrm{G}$ and $\mathrm{H})$. No significant differences in the percentage of IL-17-producing ICOS ${ }^{+}$Tregs were identified between the iRA and NC groups.

Together, these data reveal that the aRA group had increased IL-17-expressing $\mathrm{ICOS}^{+}$Tregs but decreased IL-10, TGF- $\beta$ and IL-35-expressing ICOS $^{+}$Tregs in comparison with the iRA group. Additionally, in comparison with the NCs, the aRA group had increased IL-17 and slightly increased IL-10 and IL-35, but decreased TGF- $\beta$-expressing $\mathrm{ICOS}^{+}$Tregs. Among these three groups, the iRA group had the highest IL-10, TGF- $\beta$ and IL-35-expressing ICOS ${ }^{+}$Tregs. ICOS ${ }^{+}$ Tregs may perform inflammatory and inhibitory functions by expressing IL-17, IL-10, TGF- $\beta$ and IL-35.

Cytokine expression profile prior to and following TCR stimulation are similar. TCR signaling serves several important roles in Tregs function. Given the number of abnormal $\mathrm{ICOS}^{+}$Tregs and cytokine expression in patients with RA, PBMCs were stimulated from patients with aRA and iRA, and NCs with anti-CD3 and IL-2 in vitro for 6 days. The number of $\mathrm{ICOS}^{+}$Tregs and cytokine expression were subsequently analyzed. As indicated in Fig. 4A, the number of ICOS $^{+}$Tregs was highest in the iRA group $(16.24 \pm 1.67 \%)$ and lowest in the $\mathrm{NC}$ group $(3.40 \pm 0.42 \%$; $\mathrm{P}<0.0001)$. In addition, IL-10-producing cells in $\mathrm{ICOS}^{+}$Tregs were higher in the iRA group (16.93 \pm 1.69$)$ compared with that in the aRA $(3.72 \pm 0.41$; $\mathrm{P}<0.0001)$ and $\mathrm{NC}$ groups $(0.80 \pm 0.33 ; \mathrm{P}<0.0001 ;$ Fig. $4 \mathrm{~B})$ TGF- $\beta$-producing cells in $\mathrm{ICOS}^{+}$Tregs were increased in the iRA group $(23.96 \pm 1.08)$ compared with that in the aRA (7.54 $\pm 0.80 ; \mathrm{P}<0.0001)$ and $\mathrm{NC}$ groups $(9.21 \pm 1.10 ; \mathrm{P}<0.0001$; Fig. 4C). Although the number of IL-10-producing cells in $\mathrm{ICOS}^{+}$Tregs from the iRA group was higher compared with that of the NCs, the two groups had similar numbers of TGF- $\beta$-producing cells. In comparison with suppressive cytokine expression, the number of IL-17-producing cells in $\mathrm{ICOS}^{+}$Tregs was highest in the aRA group, and no
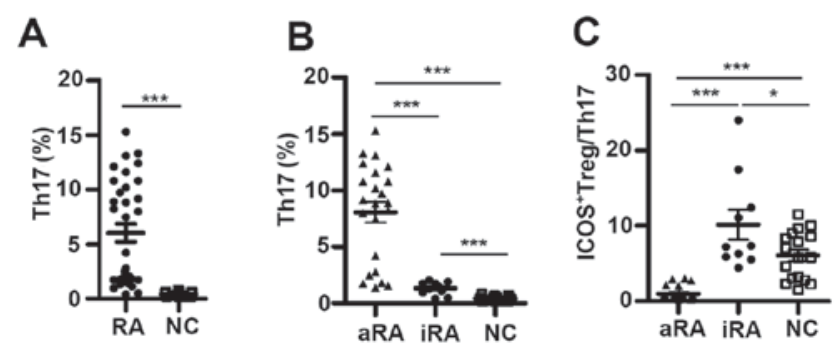

Figure 2. ICOS $^{+}$Tregs/Th17 ratios are disturbed in patients with RA. (A) The percentage of Th17 in patients with RA $(n=33)$ and in NCs $(n=17$; Mann-Whitney U test). (B) The percentage of Th17 and (C) ratio of ICOS ${ }^{+}$ Tregs/Th17 in aRA patients $(n=23)$, iRA patients $(n=10)$, and NCs $(n=17)$. Data are presented as mean \pm standard error of the mean. ${ }^{*} \mathrm{P}<0.05,{ }^{* * *} \mathrm{P}<0.0001$. ICOS, inducible T-cell costimulator; RA, rheumatoid arthritis; NCs, normal controls; aRA, active RA; iRA, inactive RA; Tregs, regulatory T cells; Th17, T helper 17 cells.

significant difference was identified between the iRA and NC groups (Fig. 4C). Thus, the differences in $\mathrm{ICOS}^{+}$Tregs observed in freshly isolated PBMCs from the three groups remained virtually unchanged following in vitro TCR and IL-2 stimulation.

\section{Discussion}

It has been reported that Th17 cells promote autoimmunity and inflammation, and diseases associated with these conditions such as RA (8), whereas Tregs are involved in maintaining immune responses and preventing autoimmunity $(11,28)$. The balance between activated responder $\mathrm{T}$ cells and Tregs may influence the extent of immunoregulation in RA (29). Additionally, humans and mice contain $\mathrm{ICOS}^{+}$and ICOS Tregs, which exhibit different properties. The current study demonstrated the amount of $\mathrm{ICOS}^{+}$Tregs increased in a cohort of Chinese patients with RA. The increase in $\mathrm{ICOS}^{+}$Tregs was larger in patients with iRA compared with those in patients with aRA, and the frequencies of $\mathrm{ICOS}^{+}$Tregs in patients with RA were negatively correlated with DAS28 scores. It was reported that IL-17, TGF- $\beta$ and IL- 6 levels detected by ELISA were increased in peripheral blood serum of patients with RA (30). Although the serum or plasma cytokines may reflect the status of disease in a patient, since several types of cells can secrete the total peripheral suppressive cytokines, the current study focused only on the cytokines produced 
A

A

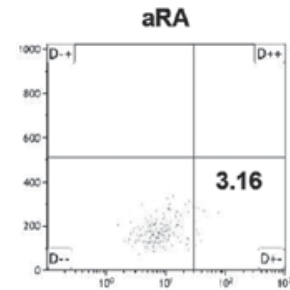

IL-10

C

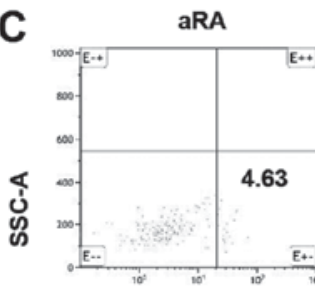

TGF- $\beta$

E

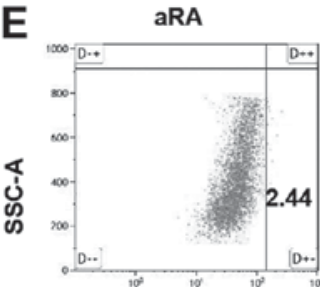

IL-35

G

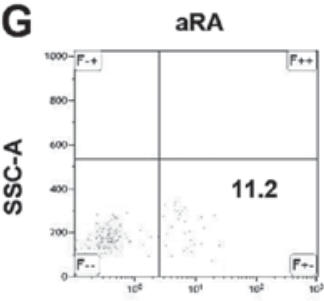

IL-17
IRA

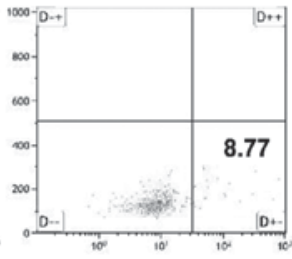

iRA
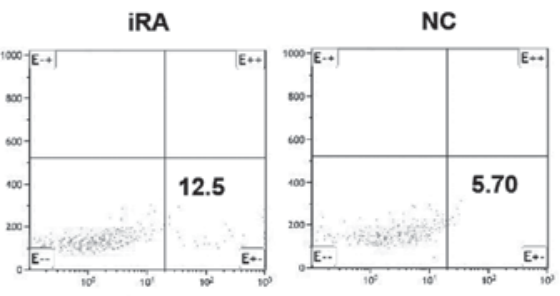

RA
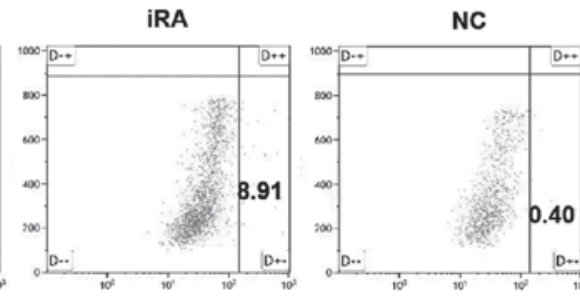

IRA
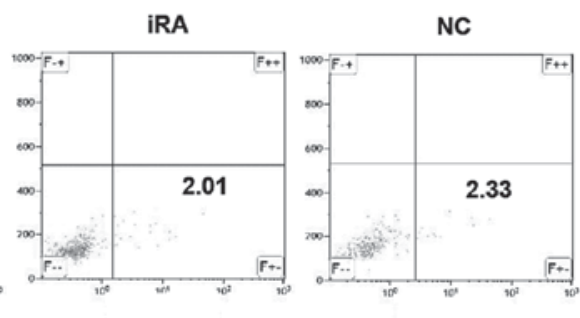

B

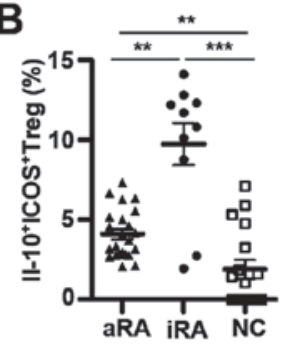

D

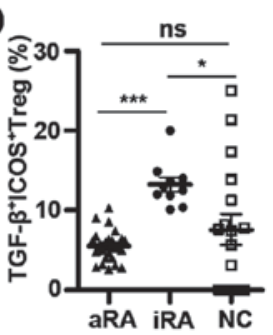

$\mathbf{F}$
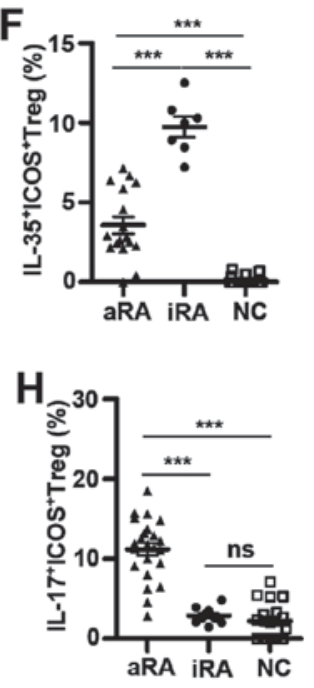

Figure 3. Anti-inflammatory cy tokines increase in RA, particularly iRA, while a pro-inflammatory cytokine increases in aRA. The numbers of cytokine-secreting ICOS+ Tregs were measured in patients with aRA ( $n=23)$, patients with iRA $(n=10)$ and NCs $(n=17)$. (A) Representative dot plots of IL-10 staining (B) and the percentage of IL- $10^{+}$ICOS $^{+}$Tregs. (C) Representative dot plots of TGF- $\beta$ staining (D) and the percentage of TGF- $\beta^{+}$ICOS ${ }^{+}$Tregs. (E) Representative dot plots of IL-35 staining and (F) the percentage of IL-35+ICOS ${ }^{+}$Tregs. (G) Representative dot plots of IL-17 staining and (H) the percentage of IL-17+ICOS ${ }^{+}$Tregs. Data are presented as mean \pm standard error of the mean. ${ }^{*} \mathrm{P}<0.05,{ }^{* *} \mathrm{P}<0.005,{ }^{* * *} \mathrm{P}<0.0005$. ICOS, inducible T-cell costimulator; RA, rheumatoid arthritis; NCs, normal controls; aRA, active RA; iRA, inactive RA; Tregs, regulatory T cells; SSC-A, side scatter area; IL, interleukin; TGF, transforming growth factor; ns, not significant.
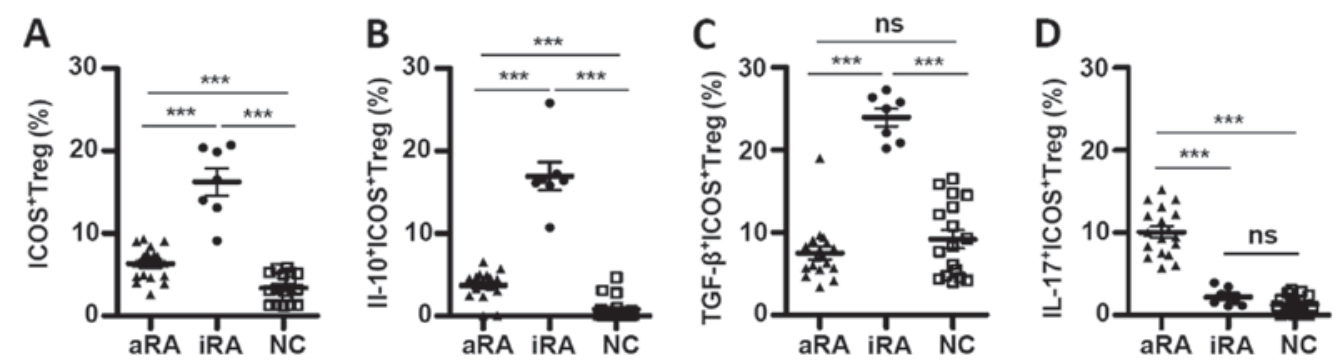

Figure 4. Cytokine expression profile prior to and following TCR stimulation are similar. PBMCs were stimulated with anti-cluster of differentiation 3 and IL-2 in vitro for 6 days. The percentage of (A) $\mathrm{ICOS}^{+}(\mathrm{B}) \mathrm{IL}-10^{+} \mathrm{ICOS}^{+}(\mathrm{C}) \mathrm{TGF}-\beta^{+} \mathrm{ICOS}{ }^{+}$and (D) $\mathrm{IL}-17^{+} \mathrm{ICOS}^{+}$in Tregs in patients with aRA ( $\mathrm{n}=18$ ), patients with iRA $(\mathrm{n}=7)$ and NCs $(\mathrm{n}=17)$. Data are presented as mean \pm standard error of the mean. ${ }^{* * * *} \mathrm{P}<0.0001$. ICOS, inducible T-cell costimulator; RA, rheumatoid arthritis; NCs, normal controls; aRA, active RA; iRA, inactive RA; Tregs, regulatory T cells; IL, interleukin; TGF, transforming growth factor; ns, not significant.

by Tregs. The data demonstrated that the $\operatorname{ICOS}^{+}$Tregs from patients with iRA expressed multiple suppressive cytokines, including IL-10, TGF- $\beta$ and IL-35, but they expressed low levels of IL-17. The patients with aRA exhibited $\mathrm{ICOS}^{+}$Tregs that expressed decreased levels of suppressive cytokines, but also had noticeably increased IL-17 levels. Data from the 
current study suggests that an abnormal number of $\mathrm{ICOS}^{+}$ Tregs and abnormal ICOS $^{+}$Treg functions may contribute to the pathogenesis of RA.

Although $\mathrm{ICOS}^{+}$cells account for a minor population within Tregs, emerging evidence has indicated that $\mathrm{ICOS}^{+}$ Tregs exhibit a strong suppressive function and that a decline in these Tregs may contribute to autoimmune disease in humans (13-16). Higher levels of ICOS expression in Tregs have been identified to correlate with improved control of autoimmune disease, diabetes and atherosclerosis $(14,18)$. The current study revealed that ICOS $^{+}$Tregs were increased in patients with RA in comparison with NCs, suggesting that the $\mathrm{ICOS}^{+}$Tregs count itself may not be the sole indicator for disease occurrence. However, patients with RA exhibit an increased number of Th17 cells, which cause decreased ICOS ${ }^{+}$ Tregs/Th17 ratios. The data from the current study suggest that imbalances between ICOS $^{+}$Tregs and Th17 may contribute to RA pathogenesis and that the $\mathrm{ICOS}^{+}$Tregs/Th17 ratio in patients with RA may be more indicative of disease progression than the $\mathrm{ICOS}^{+}$Tregs percentage itself.

Vocanson et al (31) reported that $\mathrm{CD}^{+}{ }^{+} \mathrm{CD} 25^{+} \mathrm{FoxP} 3^{+}$ $\mathrm{T}$ cells upregulated ICOS expression during in vivo sensitization, and specifically suppress hapten-reactive CD8 ${ }^{+}$ $\mathrm{T}$ cells in vivo and in vitro. The aforementioned study further demonstrated that $\mathrm{ICOS}^{+}$Tregs distinguish themselves from $\mathrm{ICOS}^{-} \mathrm{FoxP}^{+}$Tregs through their expression of IL-10, IL-17 and interferon- $\gamma$. In the current study, it was demonstrated that $\mathrm{ICOS}^{+}$Tregs exhibit a special cytokine profile characterized by the expression of inflammatory cytokine IL-17 and anti-inflammatory cytokines IL-10, TGF- $\beta$ and IL-35. Notably, ICOS ${ }^{+}$Tregs from patients with iRA exhibited more anti-inflammatory cytokines compared with patients with aRA. In contrast, $\mathrm{ICOS}^{+}$Tregs from patients with aRA expressed more IL-17 compared with those from the iRA group. This data suggests that $\mathrm{ICOS}^{+}$Tregs contribute to RA pathogenesis via at least two mechanisms. On one hand, $\mathrm{ICOS}^{+}$Tregs produce inhibitory cytokines to suppress inflammation. On the other hand, $\mathrm{ICOS}^{+}$Tregs may express proinflammatory cytokines that exacerbate RA. The balance between these two functions may dictate disease progression. Elevated pathogenic IL-17 expression and decreased suppressive cytokine-producing ICOS $^{+}$Tregs may promote disease development in patients with aRA. In contrast, predominance of suppressive cytokine-producing $\mathrm{ICOS}^{+}$Tregs over IL-17-producing $\mathrm{ICOS}^{+}$Tregs may reduce inflammation and help ensure an inactive state in patients with RA.

\section{Acknowledgements}

The authors would like to thank Dr Xiaoping Zhong (Division of Allergy and Immunology, Department of Pediatrics, Duke University Medical Center, Durham, NC27710, USA) for their helpful advice and assistance.

\section{Funding}

The present study was supported by the Nanfang Hospital Foundation of SMU (grant no. 201362) and the Guangdong Natural Science Foundation (grant no. 2017A030313508).

\section{Availability of data and materials}

The datasets used and/or analyzed during the current study are available from the corresponding author on reasonable request.

\section{Authors' contributions}

HXW, XK, WL and Y-RQ conceived and designed the experiments. XK, SC, HL, XL, XY performed the experiments. HXW, SC, XK and WL analyzed the data. HXW, XK and SC wrote the manuscript. Y-RQ had full access to all of the data in the study and takes responsibility for the integrity of the data and the accuracy of the data analysis.

\section{Ethics approval and consent to participate}

Ethics approval was received from Nanfang Hospital, Southern Medical University. All participants gave written consent during their enrollment.

\section{Patient consent for publication}

All patients gave written consent during their enrollment for publication.

\section{Competing interests}

The authors declare that they have no competing interests.

\section{References}

1. Firestein GS: Evolving concepts of rheumatoid arthritis. Nature 423: 356-361, 2003.

2. Del Junco DJ, Annegers JF, Coulam CB and Luthra HS: The relationship between rheumatoid arthritis and reproductive function. Br J Rheumatol 1(Soppl 28): 33 42-45, 1989.

3. Picerno V,Ferro F, Adinolfi A, Valentini E, Tani C and Alunno A: One year in review: The pathogenesis of rheumatoid arthritis. Clin Exp Rheumatol 33: 551-558, 2015.

4. McInnes IB and Schett G: The pathogenesis of rheumatoid arthritis. N Engl J Med 365: 2205-2219, 2011.

5. Yue C, You X, Zhao L, Wang H, Tang F, Zhang F, Zhang X and He W: The effects of adalimumab and methotrexate treatment on peripheral Th17 cells and IL-17/IL-6 secretion in rheumatoid arthritis patients. Rheumatol Int 30: 1553-1557, 2010.

6. Lu TT, Zhu P, Li XY and Fan CM: Functional status of T helper cells in rheumatoid arthritis and effect of etanercept. Xi Bao Yu Fen Zi Mian Yi Xue Za Zhi 24: 495-497, 2008 (In Chinese).

7. Miossec P: Interleukin-17 in fashion, at last: Ten years after its description, its cellular source has been identified. Arthritis Rheum 56: 2111-2125, 2007.

8. Shahrara S, Huang Q, Mandelin AN II and Pope RM: TH-17 cells in rheumatoid arthritis. Arthritis Res Ther 10: R93, 2008.

9. Campbell DJ: Control of regulatory T cell migration, function, and homeostasis. J Immunol 195: 2507-2513, 2015.

10. Byng-Maddick R and Ehrenstein MR: The impact of biological therapy on regulatory $\mathrm{T}$ cells in rheumatoid arthritis. Rheumatology (Oxford) 54: 768-775, 2015.

11. Cooles FA, Isaacs JD and Anderson AE: Treg cells in rheumatoid arthritis: An update. Curr Rheumatol Rep 15: 352, 2013.

12. Dong C, Juedes AE, Temann UA, Shresta S, Allison JP, Ruddle NH and Flavell RA: ICOS co-stimulatory receptor is essential for T-cell activation and function. Nature 409: 97-101, 2001.

13. Busse M, Krech M, Meyer-Bahlburg A, Hennig C and Hansen G: ICOS mediates the generation and function of CD4+CD25+Foxp3+ regulatory T cells conveying respiratory tolerance. J Immunol 189: 1975-1982, 2012. 
14. Herman AE, Freeman GJ, Mathis D and Benoist C: CD4+CD25+ $\mathrm{T}$ regulatory cells dependent on ICOS promote regulation of effector cells in the prediabetic lesion. J Exp Med 199: 1479-1489, 2004.

15. Chen Y, Shen S, Gorentla BK, Gao J and Zhong XP: Murine regulatory $\mathrm{T}$ cells contain hyperproliferative and death-prone subsets with differential ICOS expression. J Immunol 188: $1698-1707,2012$

16. Ito T, Hanabuchi S, Wang YH, Park WR, Arima K, Bover L, Qin FX, Gilliet M and Liu YJ: Two functional subsets of FOXP3+ regulatory $\mathrm{T}$ cells in human thymus and periphery. Immunity 28 : $870-880,2008$

17. Kohyama M, Sugahara D, Sugiyama S, Yagita H, Okumura K and Hozumi N: Inducible costimulator-dependent IL-10 production by regulatory T cells specific for self-antigen. Proc Natl Acad Sci USA 101: 4192-4197, 2004.

18. Gotsman I, Grabie N, Gupta R, Dacosta R, MacConmara M, Lederer J, Sukhova G, Witztum JL, Sharp AH and Lichtman AH: Impaired regulatory $\mathrm{T}$-cell response and enhanced atherosclerosis in the absence of inducible costimulatory molecule. Circulation 114: 2047-2055, 2006.

19. Nistala K and Wedderburn LR: Th17 and regulatory T cells: Rebalancing pro-and anti-inflammatory forces in autoimmune arthritis. Rheumatology (Oxford) 48: 602-606, 2009.

20. Niu Q, Cai B, Huang ZC, Shi YY and Wang LL: Disturbed Th17/Treg balance in patients with rheumatoid arthritis. Rheumatol Int 32: 2731-2736, 2012.

21. Wang W, Shao S, Jiao Z, Guo M, Xu H and Wang S: The Th17/Treg imbalance and cytokine environment in peripheral blood of patients with rheumatoid arthritis. Rheumatol Int 32 887-893, 2012

22. Aletaha D, Neogi T, Silman AJ, Funovits J, Felson DT, Bingham CO III, Birnbaum NS, Burmester GR, Bykerk VP, Cohen MD, et al: 2010 rheumatoid arthritis classification criteria: An american college of rheumatology/european league against rheumatism collaborative initiative. Ann Rheum Dis 69: 1580-1588, 2010.
23. Prevoo ML, van't Hof MA, Kuper HH, van Leeuwen MA, van de Putte LB and van Riel PL: Modified disease activity scores that include twenty-eight-joint counts. Development and validation in a prospective longitudinal study of patients with rheumatoid arthritis. Arthritis Rheum 38: 44-48, 1995.

24. Sakaguchi S, Yamaguchi T, Nomura T and Ono M: Regulatory T cells and immune tolerance. Cell 133: 775-787, 2008.

25. Bedoya SK, Lam B, Lau K and Larkin J III: Th17 cells in immunity and autoimmunity. Clin Dev Immunol 2013: 986789 , 2013.

26. Kirkham BW, Lassere MN, Edmonds JP, Juhasz KM, Bird PA, Lee CS, Shnier R and Portek IJ: Synovial membrane cytokine expression is predictive of joint damage progression in rheumatoid arthritis: A two-year prospective study (the DAMAGE study cohort). Arthritis Rheum 54: 1122-1131, 2006.

27. Collison LW, Workman CJ, Kuo TT, Boyd K, Wang Y, Vignali KM, Cross R, Sehy D, Blumberg RS and Vignali DA: The inhibitory cytokine IL-35 contributes to regulatory T-cell function. Nature 450: 566-569, 2007.

28. Wing K and Sakaguchi S: Regulatory T cells exert checks and balances on self tolerance and autoimmunity. Nat Immunol 11: 7-13, 2010.

29. van Amelsfort JM, Jacobs KM, Bijlsma JW, Lafeber FP and Taams LS: CD4(+)CD25(+) regulatory T cells in rheumatoid arthritis: Differences in the presence, phenotype, and function between peripheral blood and synovial fluid. Arthritis Rheum 50: 2775-2785, 2004

30. Pan F, Xiang H, Yan J, Hong L, Zhang L, Liu Y, Feng X and Cai C: Dendritic cells from rheumatoid arthritis patient peripheral blood induce Th17 cell differentiation via miR-363/Integrin $\alpha v / T G F-\beta$ axis. Scand J Immunol 85: 441-449, 2017.

31. Vocanson M, Rozieres A, Hennino A, Poyet G, Gaillard V, Renaudineau S, Achachi A, Benetiere J, Kaiserlian D, Dubois B and Nicolas JF: Inducible costimulator (ICOS) is a marker for highly suppressive antigen-specific $\mathrm{T}$ cells sharing features of TH17/TH1 and regulatory T cells. J Allergy Clin Immunol 126: 280-289, 2010 